\title{
Clinical features and death risk factors in COVID-19 patients with cancer: a retrospective study
}

\author{
Yi Zhou ${ }^{1 \dagger}$, Qiao Yang ${ }^{2 \dagger}$, Jun Ye ${ }^{3 \dagger}$, Xiaocheng Wu ${ }^{4}$, Xianhua Hou ${ }^{5}$, Yimei Feng ${ }^{6}$, Bangyu Luo ${ }^{1}$, Jixi Li ${ }^{1}$, \\ Guangrong Yang ${ }^{1}$, Lingchen $\mathrm{Li}^{1}$, Xiu Yang ${ }^{1}$, Bin Wang ${ }^{7}$, Songtao Zhao ${ }^{8}, \mathrm{Li} \mathrm{Li}^{9}, \mathrm{Qi} \mathrm{Li}^{7^{*}}, \mathrm{Zhi} \mathrm{Xu}^{7^{*}}, \mathrm{Hao} \mathrm{Wu}^{10^{*}}$ and \\ Jianguo $\operatorname{Sun}^{1 *}$ (10)
}

\begin{abstract}
Background: Coronavirus disease 2019 (COVID-19) has spread around the world. This retrospective study aims to analyze the clinical features of COVID-19 patients with cancer and identify death outcome related risk factors.

Methods: From February 10th to April 15th, 2020, 103 COVID-19 patients with cancer were enrolled. Difference analyses were performed between severe and non-severe patients. A propensity score matching (PSM) analysis was performed, including 103 COVID-19 patients with cancer and 206 matched non-cancer COVID-19 patients. Next, we identified death related risk factors and developed a nomogram for predicting the probability.

Results: In 103 COVID-19 patients with cancer, the main cancer categories were breast cancer, lung cancer and bladder cancer. Compared to non-severe patients, severe patients had a higher median age, and a higher proportion of smokers, diabetes, heart disease and dyspnea. In addition, most of the laboratory results between two groups were significantly different. PSM analysis found that the proportion of dyspnea was much higher in COVID-19 patients with cancer. The severity incidence in two groups were similar, while a much higher mortality was found in COVID-19 patients with cancer compared to that in COVID-19 patients without cancer $(11.7 \%$ vs. $4.4 \%, P=0.028)$. Furthermore, we found that neutrophil-to-lymphocyte ratio (NLR) and C-reactive protein (CRP) were related to death outcome. And a nomogram based on the factors was developed.
\end{abstract}

Conclusion: In COVID-19 patients with cancer, the clinical features and laboratory results between severe group and non-severe group were significantly different. NLR and CRP were the risk factors that could predict death outcome.

Keywords: COVID-19, Cancer, Nomogram, Neutrophil-to-lymphocyte ratio, C-reactive protein

*Correspondence: liqioliver@sina.com; xu_zhi999@163.com; xuzhihxk@tmmu.edu.cn; ewuhao@163.com; sunjg09@aliyun.com; sunjianguo@tmmu.edu.cn

${ }^{\dagger}$ Yi Zhou, Qiao Yang, Jun Ye contributed equally to this work

${ }^{1}$ Cancer Institute, Xinqiao Hospital, Army Medical University, 83 Xinqiao Zhengjie Road, Shapingba, Chongqing 400037, People's Republic of China

${ }^{7}$ Department of Respiratory and Intensive Care, Xinqiao Hospital, Army Medical University, 83 Xinqiao Zhengjie Road, Shapingba, Chongqing 400037, People's Republic of China

${ }^{10}$ Xinqiao Hospital, Army Medical University, 83 Xinqiao Zhengjie Road, Shapingba, Chongqing 400037, People's Republic of China

Full list of author information is available at the end of the article

\section{Background}

Coronavirus disease 2019 (COVID-19) has spread around the world. Patients with COVID-19 presented with fever, cough, dyspnea, vomiting and diarrhea, and the critical patients might have acute respiratory distress syndrome and multiorgan failure. Analysis of the blood test results of the patients showed that the neutrophil counts, lymphocyte counts and biochemical indexes were abnormal and the pro-inflammatory cytokines and infection-related biomarkers were highly expressed $[1,2]$. 
People with diabetes [3], hypertension [4], and heart disease [5] were thought to be susceptible to COVID-19 and had poor clinical outcome. Cancer patients were considered to have lower immunity than normal people. Therefore, they were susceptible to infected with bacteria and viruses [6, 7]. Among patients with advanced cancer, $20 \%$ had lymphopenia [8]. Therefore, immunosuppression status of cancer patients put them at an increased risk of COVID-19.

In the early stage (before February 2020) of COVID19 outbreak in Wuhan, China, medical staff and materials were inadequate, leading to a high mortality rate. By February 2020, with the arrival of a large number of medical supplies and more than 40,000 medical workers in Wuhan, the patients with COVID-19 got timely treatment, and the mortality reduced gradually [9]. Some studies reported that cancer patients were more susceptible to COVID-19 and more likely to deteriorate into a severe form [9-12]. However, most of the cases they reported were in the early stage of the outbreak, and there was a lack of knowledge and response measures of the disease. In this study, we collected cases admitted to hospital in the late stage of the outbreak in Wuhan, China, reflecting the real status of cancer patients with COVID-19 whom were admitted under adequate treatment. We aimed to determine the risk factors of disease severity and death, and sought to develop a nomogram to predict the risk.

Table 1 The categories of the 103 COVID-19 patients with cancer

\begin{tabular}{lc}
\hline Tumor types & $\begin{array}{c}\text { Number of } \\
\text { patients (\%) }\end{array}$ \\
\hline Breast cancer & $23(22.3 \%)$ \\
Lung cancer & $17(16.5 \%)$ \\
Bladder cancer & $9(8.7 \%)$ \\
Esophagus cancer & $8(7.8 \%)$ \\
Gastric cancer & $7(6.8 \%)$ \\
Thyroid cancer & $6(5.8 \%)$ \\
Rectal cancer & $5(4.9 \%)$ \\
Cervical cancer & $4(3.9 \%)$ \\
Larynx cancer & $4(3.9 \%)$ \\
Colon cancer & $4(3.9 \%)$ \\
Prostate cancer & $4(3.9 \%)$ \\
Nasopharyngeal cancer & $3(2.9 \%)$ \\
Liver cancer & $3(2.9 \%)$ \\
Endometrial cancer & $3(2.9 \%)$ \\
Testicular cancer & $1(1 \%)$ \\
Ovarian cancer & $1(1 \%)$ \\
Renal cancer & $1(1 \%)$ \\
\hline
\end{tabular}

\section{Methods}

Patients selection

This study was approved by the Ethics Committee of Taikang Hospital (TKTJLL-007). The Ethics Committee of the Taikang Hospital waived the need for informed consent of each patient. From February 10th to April 15th, 2020, a total of 2980 patients with confirmed COVID-19 infection by RT-PCR were enrolled from Taikang hospital and other temporary hospitals of Wuhan in this retrospective study, including 103 cancer patients. All patients were divided into severe or non-severe group according to the Diagnosis and Treatment Protocol for COVID-19 (Trial Version 7) published by the National Health Commission of China. The severe patients were defined as following: the respiratory rate is more than 30 breaths per minute, the oxygen saturation is lower than $93 \%$ in rest state, oxygenation index is no greater than $300 \mathrm{mmHg}$, respectively. Propensity score matching (MatchIt package, $R$, version 3.5.2) was used to match each COVID-19 patient with cancer with two COVID-19 patients without cancer for further analysis. The following predetermined factors were considered: age, gender and comorbidities, such as diabetes, hypertension and heart disease. The flow chart of the study design was shown in Additional file 1: Figure S1.

\section{Data collection and difference analyses}

Clinical features, and laboratory results of all patients with COVID-19 were obtained from the hospitals through the way of desk review of patient medical records. All data were extracted manually. Clinical features included demographics, comorbidities and symptoms. The laboratory examinations included routine blood tests, inflammation-related biomarkers, renal and liver function, biochemical tests, and coagulation function. Difference analyses between 103 COVID19 patients with cancer and 206 matched COVID-19 patients without cancer were performed. In addition, we performed difference analyses between severe and nonsevere groups, as well as between survivors and non-survivors in COVID-19 patients with cancer.

\section{Development and validation of a nomogram}

First of all, factors were excluded if missing values of the factor reached more than $20 \%$. Then, missing values were imputed using multivariate imputation if factors had missing values less than $20 \%$. Generalized linear model was used to select significant risk factors $(\mathrm{P}<0.05)$ that affect death outcome. A nomogram was developed with significant risk factors. The area under the curve (AUC) of the receiver operating characteristic (ROC) curve, calibration plot, decision curve and clinical impact curve were used to validate the nomogram. $R$ packages used in 
Table 2 Clinical features and laboratory findings differences between severe and non-severe patients

\begin{tabular}{|c|c|c|c|c|}
\hline Clinical features & All $(n=103)$ & Non-severe $(n=67)$ & Severe $(n=36)$ & P-value \\
\hline Age & $66.0(24.0-90.0)$ & $65.0(43.0-90.0)$ & $70.0(24.0-87.0)$ & 0.004 \\
\hline Gender & & & & 0.097 \\
\hline Female & $47(45.6 \%)$ & $35(52.2 \%)$ & $12(33.3 \%)$ & \\
\hline Male & $56(54.4 \%)$ & $32(47.8 \%)$ & $24(66.7 \%)$ & \\
\hline Smoking & $20(19.4 \%)$ & $8(11.9 \%)$ & $12(33.3 \%)$ & 0.017 \\
\hline \multicolumn{5}{|l|}{ Comorbidities } \\
\hline Diabetes & $14(13.6 \%)$ & $5(7.5 \%)$ & $9(25 \%)$ & 0.018 \\
\hline Hypertension & $37(35.9 \%)$ & $22(32.8 \%)$ & $15(41.7 \%)$ & 0.396 \\
\hline Heart disease & $11(10.7 \%)$ & $4(6.0 \%)$ & $7(19.4 \%)$ & 0.047 \\
\hline \multicolumn{5}{|l|}{ Symptoms } \\
\hline Fever & $73(70.9 \%)$ & $45(67.2 \%)$ & $28(77.8 \%)$ & 0.363 \\
\hline Cough & $70(68.0 \%)$ & $49(73.1 \%)$ & $21(58.3 \%)$ & 0.183 \\
\hline Dyspnea & $24(23.3 \%)$ & $9(13.4 \%)$ & $15(41.7 \%)$ & 0.003 \\
\hline Vomiting & $5(4.9 \%)$ & $2(3.0 \%)$ & $3(8.3 \%)$ & 0.340 \\
\hline Diarrhea & $7(6.8 \%)$ & $3(4.5 \%)$ & $4(11.1 \%)$ & 0.235 \\
\hline \multicolumn{5}{|l|}{ Laboratory findings } \\
\hline WBC $\left(\times 10^{9} / \mathrm{L}\right)$ & $5.29(1.90-25.10) ; n=100$ & $4.77(1.90-25.10) ; n=65$ & $7.16(2.00-18.80) ; n=35$ & $<0.001$ \\
\hline Neutrophil $\left(\times 10^{9} / \mathrm{L}\right)$ & $3.49(0.67-22.40) ; n=100$ & $3.00(0.67-22.40) ; n=65$ & $5.27(1.03-17.36) ; n=35$ & $<0.001$ \\
\hline Lymphocyte (× 109/L) & $1.06(0.22-2.89) ; n=100$ & $1.23(0.33-2.62) ; n=65$ & $0.80(0.22-2.89) ; n=35$ & 0.003 \\
\hline NLR & $2.93(0.64-32.23) ; n=100$ & $2.29(0.64-18.39) ; n=65$ & $6.93(1.20-32.23) ; n=35$ & $<0.001$ \\
\hline Monocytes $\left(\times 10^{9} / \mathrm{L}\right)$ & $0.43(0.11-9.90) ; n=72$ & $0.41(0.11-9.90) ; n=52$ & $0.50(0.14-1.04) ; n=20$ & 0.297 \\
\hline Eosnophils $\left(\times 10^{9} / \mathrm{L}\right)$ & $0.07(0.00-0.90) ; n=72$ & $0.08(0.00-0.51) ; n=52$ & $0.03(0.00-0.90) ; n=20$ & 0.007 \\
\hline Basophils $\left(\times 10^{9} / \mathrm{L}\right)$ & $0.02(0.00-0.11) ; n=72$ & $0.02(0.00-0.11) ; n=52$ & $0.02(0.00-0.05) ; n=20$ & 0.09 \\
\hline $\mathrm{RBC}\left(\times 10^{12} / \mathrm{L}\right)$ & $3.95(2.03-5.77) ; n=73$ & $4.01(2.03-5.14) ; n=52$ & $3.53(2.63-5.77) ; n=21$ & 0.116 \\
\hline $\mathrm{HB}(\mathrm{g} / \mathrm{L})$ & $116.00(68.00-154.00) ; n=73$ & $119.00(68.00-154.00) ; n=52$ & $102.00(79.00-154.00) ; n=21$ & 0.065 \\
\hline $\operatorname{PLT}\left(\times 10^{9} / \mathrm{L}\right)$ & $203.00(53.00-431.00) ; n=72$ & $205.00(67.00-431.00) ; n=52$ & $190.00(53.00-312.00) ; n=20$ & 0.606 \\
\hline $\mathrm{CRP}(\mathrm{mg} / \mathrm{L})$ & $2.52(0.14-280.32) ; n=90$ & $1.39(0.14-137.52) ; n=58$ & $42.59(0.05-280.32) ; n=32$ & $<0.001$ \\
\hline $\mathrm{ALT}(\mathrm{U} / \mathrm{L})$ & $19.17(4.50-131.20) ; n=97$ & $19.05(4.50-131.20) ; n=64$ & $20.70(6.00-100.30) ; n=33$ & 0.87 \\
\hline AST (U/L) & $21.70(10.00-147.20) ; n=84$ & $20.45(10.00-147.20) ; n=52$ & $26.70(12.00-92.60) ; n=32$ & 0.014 \\
\hline Total protein (g/L) & 64.99 (53.70-87.58); $n=71$ & $65.50(55.45-87.58) ; n=51$ & $60.89(53.70-78.97) ; n=20$ & 0.04 \\
\hline Albumin $(\mathrm{g} / \mathrm{L})$ & $37.29(23.60-48.00) ; n=71$ & $38.14(23.60-48.00) ; n=51$ & $32.61(25.30-43.50) ; n=20$ & 0.002 \\
\hline Globulin (g/L) & $28.00(17.31-46.24) ; n=71$ & $27.30(17.31-40.49) ; n=51$ & $28.20(24.00-46.24) ; n=20$ & 0.818 \\
\hline $\mathrm{A} / \mathrm{G}$ & $1.30(0.39-2.20) ; n=71$ & $1.35(0.39-2.20) ; n=51$ & $1.22(0.71-1.46) ; n=20$ & 0.017 \\
\hline Total bilirubin (umol/L) & $10.32(4.20-44.58) ; n=70$ & $10.23(4.20-33.46) ; n=51$ & $11.70(6.00-44.58) ; n=19$ & 0.152 \\
\hline Direct Bilirubin (umol/L) & $2.71(0.00-23.90) ; n=70$ & $2.50(0.00-14.20) ; n=51$ & $3.86(0.00-23.90) ; n=19$ & 0.053 \\
\hline Indirect bilirubin (umol/L) & $7.39(2.99-26.42) ; n=70$ & $7.32(3.00-26.42) ; n=51$ & $7.90(2.99-22.68) ; n=19$ & 0.468 \\
\hline BUN (umol/L) & $4.67(2.68-29.61) ; n=68$ & $4.62(2.68-29.61) ; n=49$ & $5.47(2.93-18.77) ; n=19$ & 0.114 \\
\hline Creatinine (umol/L) & $57.72(13.25-345.35) ; n=70$ & $58.17(31.60-345.35) ; n=50$ & 55.15 (13.25-145.39); $n=20$ & 0.559 \\
\hline Uric acid (umol/L) & $268.01(100.00-275.95)) ; n=69$ & $304.73(151.56-575.95) ; n=50$ & $163.00(100.00-547.00) ; n=19$ & $<0.001$ \\
\hline $\operatorname{ALP}(\mathrm{U} / \mathrm{L})$ & $71.10(42.59-493.30) ; n=70$ & $67.80(42.59-493.30) ; n=51$ & $74.60(55.16-238.00) ; n=19$ & 0.212 \\
\hline$\gamma-\mathrm{GT}(\mathrm{U} / \mathrm{L})$ & $26.47(8.15-263.60) ; n=70$ & $26.30(8.15-263.60) ; n=51$ & $33.62(11.02-239.60) ; n=19$ & 0.262 \\
\hline CK $(U / L)$ & $48.40(10.90-195.90) ; n=55$ & $52.67(16.89-116.73) ; n=37$ & $43.14(10.90-195.90) ; n=18$ & 0.244 \\
\hline CKMB (U/L) & $7.80(0.01-54.60) ; n=68$ & $7.61(0.01-54.60) ; n=50$ & $7.85(2.28-49.70) ; n=18$ & 0.416 \\
\hline $\mathrm{LDH}(\mathrm{U} / \mathrm{L})$ & $179.90(2.17-761.70) ; n=69$ & $169.13(2.17-761.70) ; n=50$ & $209.66(116.20-702.30) ; n=19$ & 0.001 \\
\hline $\mathrm{a}-\mathrm{HBDH}(\mathrm{U} / \mathrm{L})$ & $133.59(87.75-701.40) ; n=66$ & $126.96(87.75-701.40) ; n=48$ & $169.50(99.79-604.40) ; n=18$ & 0.005 \\
\hline D-dimer (mg/L) & $0.43(0.02-27.94) ; n=45$ & $0.37(0.07-4.97) ; n=30$ & $1.14(0.02-27.94) ; n=15$ & 0.001 \\
\hline NT-proBNP (U/L) & $30.77(0.01-515.18) ; n=22$ & $11.33(0.01-112.76) ; n=11$ & $60.90(23.94-515.81) ; n=11$ & 0.001 \\
\hline Procalcitonin (ng/ml) & $0.05(0.02-0.90) ; n=56$ & $0.04(0.02-0.45) ; n=36$ & $0.18(0.02-0.90) ; n=20$ & $<0.001$ \\
\hline IL-6 (pg/ml) & $3.64(1.50-3392.00) ; n=50$ & $2.84(1.50-268.30) ; n=33$ & $29.99(1.50-3392.00) ; n=17$ & 0.001 \\
\hline
\end{tabular}


Table 2 (continued)

WBC white blood cell, NLR leukocyte to lymphocyte ratio, $R B C$ red blood cell, $H B$ hemoglobin, $P L T$ platelet, CRP C-reactive protein, $A L T$ alanine transaminase, AST aspartate transaminase, $A / G$ albumin to globulin ratio, $B U N$ blood urea nitrogen, $A L P$ alkaline phosphatase, $\gamma-G T$ gamma-glutamyl transpeptidase, $C K$ creatine kinase, $C K M B M B$ isoenzyme of creatine kinase, $L D H$ lactate dehydrogenase, $a-H B D H$ alpha-hydroxybutyric dehydrogenase, NT-proBNP N-terminal pro brain natriuretic peptide, IL-6 interleukin-6

this step including MICE, pROC, rmda, regplot, rms and PredictABEL (version 3.5.2).

\section{Statistical analysis}

Continuous variables were expressed by median and interquartile range (IQR), and categorical variables were represented as frequencies. All statistical analyses were performed using SPSS (version 17.0). The Mann-Whitney $U$ test was used to compare continuous data and Pearson's $\mathrm{X}^{2}$ test used to compare contingency data. A two-sided $\mathrm{P}$ value $<0.05$ was considered statistically significant.

\section{Results}

\section{Cancer distribution in 103 COVID-19 patients}

A total of 103 (3.5\%) cancer cases in 2980 patients with COVID-19 were enrolled in this study. The cancer categories of the 103 patients included breast cancer (22.3\%), lung cancer (16.5\%), bladder cancer (8.7\%), esophageal cancer $(7.8 \%)$, gastric cancer $(6.8 \%)$, thyroid cancer (5.8\%), rectal cancer (4.9\%), colon cancer (3.9\%), larynx cancer $(3.9 \%)$, cervical cancer $(3.9 \%)$, prostate cancer (3.9\%), liver cancer (2.9\%), nasopharyngeal cancer (2.9\%), endometrial cancer (2.9\%), renal cancer $(1 \%)$, ovarian cancer $(1 \%)$ and testicular cancer (1\%) (Table1).

\section{Comparison between severe and non-severe patients}

The patients consisted of 47 females (45.6\%) and 56 males $(54.4 \%)$, and $36(35 \%)$ in severe group and $67(65 \%)$ in non-severe group. The median age of cancer patients was 66 (ranging from 24 to 90), and the age in the severe group was older than that in non-severe group. The smoking people accounted for a higher proportion in the severe group than in the non-severe group (Table 2).

There were $42(40.8 \%)$ patients who had comorbidities, such as hypertension (35.9\%), diabetes (13.6\%), and heart disease (10.7\%), 14 of whom had two or more comorbidities. Among the 36 severe patients, 18 (50\%) cases had comorbidities, while in the 67 non-severe patients 24 (35.8\%) had comorbidities. A higher proportion of patients with diabetes or heart disease were observed in the severe group than in the non-severe group (Table 2).

The common clinical symptoms of cancer patients were fever (70.9\%), cough (68\%), dyspnea (23.3\%), diarrhea (6.8\%) and vomiting (4.9\%). The percentage of patients with cough, vomiting or diarrhea showed no significant difference between the two groups except for a higher percentage of patients had dyspnea in the severe group than in the non-severe group ( $41.7 \%$ vs. $13.4 \%, \mathrm{P}=0.003)$ (Table 2).

The median level of white blood cell (WBC) count was $5.29 \times 10^{9} / \mathrm{L}$ in all cancer patients, $4.77 \times 10^{9} / \mathrm{L}$ in nonsevere patients and $7.16 \times 10^{9} / \mathrm{L}$ in severe patients. Similar results were found for neutrophil count. The median level of lymphocyte count was significantly lower in the severe group than in the non-severe group $\left(0.80 \times 10^{9}\right.$ $/ \mathrm{L}$ vs. $\left.1.23 \times 10^{9} / \mathrm{L}, \mathrm{P}=0.003\right)$. In addition, a higher neutrophil-to-lymphocyte ratio (NLR) in severe patients was found $(6.93$ vs. $2.29, \mathrm{P}<0.001)$. The level of procalcitonin, C-reactive protein (CRP) and interleukin-6 (IL-6) was higher in severe patients than in non-severe patients (Table 2). When comparing hepatic function, renal function and biochemical indexes between severe and non-severe patients with cancer, we found that lactate dehydrogenase (LDH), alpha-hydroxybutyric dehydrogenase $(\alpha \mathrm{HBDH}), \mathrm{D}$-dimer, $\mathrm{N}$-terminal pro brain natriuretic peptide (NTproBNP), aspartate transaminase (AST) and Uric acid were higher while total protein, albumin and albumin/globulin were lower in the severe group (Table 2).

\section{Propensity score matching analysis}

We performed a 1:2 (COVID-19 with cancer: COVID19 without cancer) matched case-control analysis. Age, gender and comorbidities were used for matching. Then, differences in demographics, comorbidities, symptoms, severity, clinical outcome and laboratory findings were compared (Table 3). We found that COVID-19 patients with cancer had a higher proportion of dyspnea than COVID-19 patients without cancer $(23.3 \%$ vs. $10.2 \%$, $\mathrm{P}=0.003)$. No other significant differences were found. When comparing laboratory results between two groups, we found that the median lymphocyte count, monocytes count, eosinophils count and alanine transaminase (ALT) were lower in COVID-19 patients with cancer than in COVID-19 patients without cancer (Table 3). In contrast, the median NLR was higher in COVID-19 patients with cancer compared to counterpart. No significant differences were found in other laboratory results. The proportion of severe patients was similar between two groups ( $35 \%$ vs. $31 \%, \mathrm{P}=0.520$ ). The proportion of deaths was much higher in COVID-19 patients with cancer than 
Table 3 Comparison between COVID-19 patients with cancer and without cancer

\begin{tabular}{|c|c|c|c|c|}
\hline Clinical features & All patients $(n=309)$ & Non-cancer patients $(n=206)$ & Cancer patients $(n=103)$ & P-value \\
\hline Age & $66.0(20.0-95.0)$ & $66.00(20.00-95.00)$ & $66.0(24.0-90.0)$ & 0.948 \\
\hline Gender & & & & 0.810 \\
\hline Female & $138(44.7 \%)$ & $91(44.1 \%)$ & $47(45.6 \%)$ & \\
\hline Male & $171(55.3 \%)$ & $115(55.8 \%)$ & $56(54.4 \%)$ & \\
\hline \multicolumn{5}{|l|}{ Comorbidities } \\
\hline Diabetes & $37(11.9)$ & $23(11.1 \%)$ & $14(13.6 \%)$ & 0.579 \\
\hline Hypertension & $96(31.0 \%)$ & $59(28.6 \%)$ & $37(35.9 \%)$ & 0.195 \\
\hline Heart disease & $41(13.2)$ & $30(14.5 \%)$ & $11(10.7 \%)$ & 0.379 \\
\hline \multicolumn{5}{|l|}{ Symptoms } \\
\hline Fever & $234(74.7 \%)$ & $161(78.1)$ & $73(70.9 \%)$ & 0.163 \\
\hline Cough & $207(66.9 \%)$ & $137(66.5 \%)$ & $70(68.0 \%)$ & 0.898 \\
\hline Dyspnea & $45(14.6 \%)$ & $21(10.2 \%)$ & $24(23.3 \%)$ & 0.003 \\
\hline Vomiting & $10(3.2 \%)$ & $5(2.4 \%)$ & $5(4.9 \%)$ & 0.310 \\
\hline Diarrhoea & $17(5.5)$ & $10(4.8 \%)$ & $7(6.8 \%)$ & 0.597 \\
\hline Severity & & & & 0.520 \\
\hline Non-severe & $209(67.6 \%)$ & $142(68.9 \%)$ & $67(65 \%)$ & \\
\hline Severe & $100(32.4 \%)$ & $64(31 \%)$ & $36(35 \%)$ & \\
\hline Clinical outcome & & & & 0.028 \\
\hline Survivor & $288(93.2 \%)$ & $197(95.6 \%)$ & $91(88.3 \%)$ & \\
\hline Non-survivor & $21(6.8 \%)$ & $9(4.4 \%)$ & $12(11.7 \%)$ & \\
\hline \multicolumn{5}{|l|}{ Laboratory findings } \\
\hline WBC $(\times 109 / L)$ & $5.50(0.80-28.20) ; n=306$ & $5.60(0.80-28.2) ; n=206$ & $5.29(1.90-25.10) ; n=100$ & 0.381 \\
\hline Neutrophil (× 109/L) & $3.25(0.44-26.07) ; n=306$ & $3.23(0.44-26.07) ; n=206$ & $3.49(0.67-22.40) ; n=100$ & 0.164 \\
\hline Lymphocyte (× 109/L) & $1.41(0.60-3.50) ; n=306$ & $1.54(0.60-3.50) ; n=206$ & $1.06(0.22-2.89) ; n=100$ & $<0.001$ \\
\hline NLR & $2.28(0.20-62.12) ; n=306$ & $2.11(0.20-62.12) ; n=206$ & $2.93(0.64-32.23) ; n=100$ & $<0.001$ \\
\hline Monocytes (× 109/L) & $0.48(0.03-1.16) ; n=278$ & $0.50(0.03-1.16) ; n=206$ & $0.43(0.11-1.11) ; n=72$ & 0.040 \\
\hline Eosnophils (× 109/L) & $0.11(0.00-0.90) ; n=278$ & $0.12(0.00-0.72) ; n=206$ & $0.07(0.00-0.90) ; n=72$ & $<0.001$ \\
\hline Basophils (× 109/L) & $0.03(0.00-0.11) ; n=278$ & $0.03(0.00-0.08) ; n=206$ & $0.02(0.00-0.11) ; n=72$ & 0.076 \\
\hline $\mathrm{RBC}(\times 1012 / \mathrm{L})$ & $3.93(1.17-5.77) ; n=279$ & $3.93(1.17-5.60) ; n=206$ & $3.95(2.03-5.77) ; n=73$ & 0.406 \\
\hline $\mathrm{HB}(\mathrm{g} / \mathrm{L})$ & $120.00(53.00-166.00) ; n=279$ & $120.50(53.00-166.00) ; n=206$ & $116.00(68.00-154.00) ; n=73$ & 0.074 \\
\hline $\mathrm{PLT}(\times 109 / \mathrm{L})$ & $209.50(4.00-443.00) ; n=278$ & $213.00(4.00-443.00) ; n=206$ & $203.00(53.00-431.00) ; n=72$ & 0.120 \\
\hline $\mathrm{CRP}(\mathrm{mg} / \mathrm{L})$ & $2.27(0.01-280.32) ; n=251$ & $2.16(0.01-268.60) ; n=161$ & $2.52(0.14-280.32) ; n=90$ & 0.147 \\
\hline $\mathrm{ALT}(\mathrm{U} / \mathrm{L})$ & $21.67(4.50-239.70) ; n=301$ & $23.35(5.30-239.70) ; n=204$ & $19.17(4.50-131.20) ; n=97$ & 0.047 \\
\hline $\mathrm{AST}(\mathrm{U} / \mathrm{L})$ & $21.55(9.60-502.40) ; n=260$ & $21.51(9.60-502.40) ; n=176$ & $21.70(10.00-147.20) ; n=84$ & 0.374 \\
\hline Total protein (g/L) & $65.5(32.70-87.58) ; n=276$ & $65.81(32.70-81.6) ; n=205$ & $64.99(53.70-87.58) ; n=71$ & 0.528 \\
\hline Albumin (g/L) & $37.30(18.3-48.00) ; n=276$ & $37.36(18.30-45.55) ; n=205$ & $37.29(23.60-48.00) ; n=71$ & 0.723 \\
\hline Globulin (g/L) & $27.73(14.40-46.24) ; n=276$ & $27.70(14.40-45.91) ; n=205$ & $28.00(17.31-46.24) ; n=71$ & 0.417 \\
\hline $\mathrm{A} / \mathrm{G}$ & $1.33(0.39-2.65) ; n=276$ & $1.33(0.70-2.65) ; n=205$ & $1.30(0.39-2.20) ; n=71$ & 0.338 \\
\hline Total bilirubin (umol/L) & $10.00(4.20-415.70) ; n=274$ & $9.76(4.20-415.70) ; n=204$ & $10.32(4.20-44.58) ; n=70$ & 0.514 \\
\hline Direct Bilirubin (umol/L) & $2.50(0.00-326.70) ; n=274$ & $2.50(0.00-326.70) ; n=204$ & $2.71(0.00-23.90) ; n=70$ & 0.412 \\
\hline Indirect bilirubin (umol/L) & $7.18(2.69-89.01) ; n=274$ & $7.17(2.69-89.01) ; n=204$ & $7.39(2.99-26.42) ; n=70$ & 0.883 \\
\hline BUN (umol/L) & $4.88(2.23-36.08) ; n=272$ & $4.93(2.23-36.08) ; n=204$ & $4.67(2.68-29.61) ; n=68$ & 0.471 \\
\hline Creatinine (umol/L) & $60.00(13.25-345.35) ; n=273$ & $60.87(26.77-243.40) ; n=203$ & $57.72(13.25-345.35) ; n=70$ & 0.509 \\
\hline Uric acid (umol/L) & $281.63(69.00-600.56) ; n=273$ & $286.02(69.00-600.56) ; n=204$ & $268.01(100.00-575.95) ; n=69$ & 0.274 \\
\hline $\mathrm{ALP}(\mathrm{U} / \mathrm{L})$ & 71.10 (31.90-493.30); n=272 & $71.12(31.90-366.10) ; n=202$ & $71.10(42.59-493.30) ; n=70$ & 0.461 \\
\hline $\mathrm{Y}-\mathrm{GT}(\mathrm{U} / \mathrm{L})$ & $26.50(7.64-263.6) ; n=273$ & $26.50(7.64-242.2) ; n=203$ & $26.47(8.15-263.60) ; n=70$ & 0.813 \\
\hline CK (U/L) & $44.31(10.90-1210.00) ; n=229$ & $43.20(11.00-1210.00) ; n=174$ & $48.40(10.90-195.90) ; n=55$ & 0.084 \\
\hline $\mathrm{LDH}(\mathrm{U} / \mathrm{L})$ & 175.35 (2.17-1489.89); n= 270 & 174.10 (99.90-489.89); n= 201 & $179.90(2.17-761.70) ; n=69$ & 0.686 \\
\hline $\mathrm{a}-\mathrm{HBDH}(\mathrm{U} / \mathrm{L})$ & $130.30(79.66-1028.00) ; n=267$ & $129.80(79.66-1028.00) ; n=201$ & $133.59(87.75-701.40) ; n=66$ & 0.511 \\
\hline
\end{tabular}


Table 3 (continued)

\begin{tabular}{|c|c|c|c|c|}
\hline Clinical features & All patients $(n=309)$ & Non-cancer patients $(n=206)$ & Cancer patients $(n=103)$ & P-value \\
\hline D-dimer (mg/L) & $0.44(0.01-27.94) ; n=172$ & $0.44(0.01-6.91) ; n=127$ & $0.43(0.02-27.94) ; n=45$ & 0.498 \\
\hline NT-proBNP (U/L) & $43.94(0.01-35,000.00) ; n=102$ & $51.61(0.01-35,000.00) ; n=80$ & $30.77(0.01-515.18) ; n=22$ & 0.503 \\
\hline Procalcitonin (ng/ml) & $0.045(0.01-87.04) ; n=200$ & $0.042(0.01-87.04) ; n=144$ & $0.05(0.02-0.90) ; n=56$ & 0.231 \\
\hline IL-6 (pg/ml) & $3.20(1.50-5000.00) ; n=189$ & $3.0(1.50-5000.00) ; n=139$ & $3.64(1.50-3392.00) ; n=50$ & 0.099 \\
\hline
\end{tabular}

WBC white blood cell, NLR leukocyte to lymphocyte ratio, RBC red blood cell, $H B$ hemoglobin, $P L T$ platelet, $C R P C$-reactive protein, $A L T$ alanine transaminase, $A S T$ aspartate transaminase, $A / G$ albumin to globulin ratio, $B U N$ blood urea nitrogen, $A L P$ alkaline phosphatase, $\gamma$-GT gamma-glutamyl transpeptidase, $C K$ creatine kinase, CKMB MB isoenzyme of creatine kinase, $L D H$ lactate dehydrogenase, $a-H B D H$ alpha-hydroxybutyric dehydrogenase, NT-proBNP N-terminal pro brain natriuretic peptide, IL-6 interleukin- 6

Table 4 Comparison between survivors and non-survivors in COVID-19 patients with cancer

\begin{tabular}{|c|c|c|c|c|}
\hline Clinical features & All patients $(n=103)$ & Survivor $(n=91)$ & Non-survivor $(n=12)$ & P-value \\
\hline Age & $66.0(24.0-90.0)$ & $66(24.0-90.0)$ & $66(56.0-81.0)$ & 0.487 \\
\hline Gender & & & & 0.061 \\
\hline Female & $47(45.6 \%)$ & $45(44.6 \%)$ & $2(16.7 \%)$ & \\
\hline Male & $56(54.4 \%)$ & $56(55.4 \%)$ & $10(83.3 \%)$ & \\
\hline Smoking & $20(19.4 \%)$ & $16(17.6 \%)$ & $4(33.3 \%)$ & 0.242 \\
\hline \multicolumn{5}{|l|}{ Comorbidities } \\
\hline Diabetes & $14(13.6 \%)$ & $13(14.3 \%)$ & $1(8.3 \%)$ & 1.000 \\
\hline Hypertension & 37 (35.9\%) & $36(39.6 \%)$ & $1(8.3 \%)$ & 0.052 \\
\hline Heart disease & $11(10.7 \%)$ & $9(9.9 \%)$ & $2(16.7 \%)$ & 0.613 \\
\hline \multicolumn{5}{|l|}{ Symptoms } \\
\hline Fever & $73(70.9 \%)$ & $63(69.2 \%)$ & $10(83.3 \%)$ & 0.501 \\
\hline Cough & 70 (68.0\%) & $63(69.2 \%)$ & $7(58.3 \%)$ & 0.183 \\
\hline Dyspnea & $24(23.3 \%)$ & $16(17.6 \%)$ & $8(66.7 \%)$ & 0.003 \\
\hline Vomiting & $5(4.9 \%)$ & $4(4.4 \%)$ & $1(8.3 \%)$ & 0.34 \\
\hline Diarrhoea & $7(6.8 \%)$ & $6(6.7 \%)$ & $1(8.3 \%)$ & 0.235 \\
\hline \multicolumn{5}{|l|}{ Laboratory findings } \\
\hline WBC $(\times 109 / L)$ & $5.29(1.90-25.10) ; n=100$ & $5.09(1.90-25.10) ; n=88$ & $7.36(3.50-18.80) ; n=12$ & $<0.001$ \\
\hline Neutrophil (× 109/L) & $3.49(0.67-22.40) ; n=100$ & $3.25(0.67-22.40) ; n=88$ & $6.85(1.50-17.36) ; n=12$ & $<0.001$ \\
\hline Lymphocyte (× 109/L) & $1.06(0.22-2.89) ; n=100$ & $1.15(0.33-2.89) ; n=88$ & $0.64(0.22-2.40) ; n=12$ & 0.012 \\
\hline NLR & $2.93(0.64-32.23) ; n=100$ & $2.64(0.64-24.76) ; n=88$ & $11.7(2.88-32.32) ; n=12$ & $<0.001$ \\
\hline $\mathrm{CRP}(\mathrm{mg} / \mathrm{L})$ & $2.52(0.14-280.32) ; n=90$ & $1.79(0.14-177.80) ; n=81$ & $80.00(4.80-280.32) ; n=9$ & $<0.001$ \\
\hline $\mathrm{ALT}(\mathrm{U} / \mathrm{L})$ & $19.17(4.50-131.20) ; n=97$ & $19.17(4.50-131.20) ; n=85$ & $16.50(6.00-68.30) ; n=12$ & 0.634 \\
\hline $\mathrm{AST}(\mathrm{U} / \mathrm{L})$ & $21.70(10.00-147.20) ; n=84$ & $21.20(10.00-147.20) ; n=72$ & $31.35(12.00-92.6) ; n=12$ & 0.164 \\
\hline
\end{tabular}

WBC white blood cell, NLR leukocyte to lymphocyte ratio, CRP C-reactive protein, ALT alanine transaminase, AST Aspartate transaminase

in COVID-19 patients without cancer $(11.7 \%$ vs. $4.4 \%$, $\mathrm{P}=0.028$ ) (Table 3).

\section{Nomogram for predicting death outcome}

As of April 15th, 2020, 12 out of 103 COVID-19 patients with cancer died in this study. To identify risk factors for death outcome and develop a nomogram, we excluded factors if the missing values were more than $20 \%$. Hence, age, gender, smoking, comorbidities, symptoms, WBC count, Neutrophil count, Lymphocyte count, NLR, CRP, ALT and AST were included for further analysis. Comparison between survivors and non-survivors were performed. As shown in Table 4, when comparing clinical features, a higher proportion of dyspnea was found in non-survivors. No significant differences were found in other clinical features between two groups. When comparing laboratory results, we found that WBC count, neutrophil count, NLR and CRP were higher in non-survivors, while lymphocyte count was lower in non-survivors.

Age, gender, smoking, number of comorbidities, number of symptoms, WBC, NLR, CRP, ALT, and AST were included in the generalized linear model. The results indicated that NLR and CRP were significant risk factors 
Table 5 P-value of each factor in the generalized linear model

\begin{tabular}{lll}
\hline Factors & Death outcome & Severe outcome \\
\hline Age & 0.990 & 0.514 \\
Sex & 0.130 & 0.340 \\
Smoking & 0.897 & 0.097 \\
Number of comorbidities & 0.130 & 0.279 \\
Number of symptoms & 0.565 & 0.552 \\
WBC & 0.318 & 0.469 \\
NLR & 0.033 & 0.040 \\
CRP & 0.040 & 0.082 \\
ALT & 0.588 & 0.451 \\
AST & 0.947 & 0.706 \\
\hline
\end{tabular}

WBC white blood cell, $N L R$ leukocyte to lymphocyte ratio, CRP C-reactive protein, $A L T$ alanine transaminase, AST aspartate transaminase

related to death outcome (Table 5). A nomogram to predict the probability of death was developed based on the two factors (Fig. 1a). The AUC of the nomogram was 0.918 (95\% confidence interval [CI] 0.860-0.977), which was higher than that of NLR $(0.872,95 \%$ CI $0.784-0.961)$ and CRP $(0.880,95 \%$ CI $0.803-0.958)$ (Fig. 1b). The calibration plot showed that the predicted outcomes were in high agreement with observed outcomes (Fig. 1c). The decision curve and clinical impact curve showed that the nomogram had superior net benefit and influence on the death outcome of patients (Fig. 1d-e).

For severe outcome, only NLR was significant factor in the generalized linear model (Table 5), hence we didn't develop a nomogram for predicting the probability of severity.

\section{Discussion}

The World Health Organization had declared COVID19 to be a public health emergency of the world [11]. Patients with cancer are at higher risk of COVID-19, because they have low immunity and myelosuppressive effects caused by chemotherapy, surgery or radiotherapy for the cancer. Some studies reported that about 1-5.6\% COVID-19 patients had cancer [9, 10, 13, 14]. In our study, we found that approximately 3.5\% COVID-19 patients had cancer, which was consistent with that in previous reports.

A multicentre cohort study from China published earlier reported that COVID-19 patients with cancer had 24 different types of cancer, with breast cancer, colorectal cancer and lung cancer being the most common types [9]. Another small cohort study from China found that the most common cancer with COVID-19 was lung cancer, followed by breast cancer and rectal cancer [10]. In this study, we reported a total of 17 different types of cancer, of which breast cancer, lung cancer and bladder cancer were the three most common types of cancer.

The main symptoms of COVID-19 patients with cancer were fever, cough, dyspnea, vomiting, diarrhea, and so on, which were similar to those without cancer. When comparing cancer patients with severe COVID-19 with cancer patients with non-severe COVID-19, we found that the severe group had a higher median age and a higher proportion of smoker, diabetes, heart disease and dyspnea. Blood tests can real-time indicate the patient's condition. In severe group, the WBC count and neutrophil count were higher and lymphocyte count was lower, so the NLR is a significant factor to predict the severity of patients. We also found that the levels of pro-inflammatory cytokines and infection-related biomarkers were higher in severe COVID-19 patients with cancer, including procalcitonin, CRP and IL-6. In addition, the COVID19 might injure the liver, heart, kidney and other vital organs. Hence, LDH, $\alpha-\mathrm{HBDH}, \mathrm{D}$-dimer, NTproBNP, AST were higher in severe COVID-19 patients with cancer.

Wang and colleagues reported a much higher proportion of severe COVID-19 in cancer patients than in noncancer patients [9]. But our study found that the severity rate in cancer patients was similar to that in non-cancer patients. The reason may be that the patients in our study were admitted to hospital after February 2020 and the patients reported in those studies were admitted to hospital mostly before February 2020. Patients included in this study received better treatment because of sufficient medical resources. So the severe rate of cancer patients in this study was lower than that in previous reports.

The mortality in COVID-19 patients with cancer ranged from 15 to $28.6 \%$ in previous studies $[9,10,15]$. In this study, we reported a mortality of $11.7 \%$ in COVID19 patients with cancer, which was higher than that in COVID-19 patients without cancer (4.4\%). The global outbreak of COVID-19 brings huge challenges to the rational use of medical resources. Therefore, how to use effective markers to screen patients who need intensive care or have a high risk of death will help allocate medical resources effectively and reasonably, as well as reduce the mortality. Liang and his colleagues found that X-ray abnormality, age, hemoptysis, dyspnea, unconsciousness, number of comorbidities, cancer history, NLR, LDH and direct bilirubin at admission were significant predictors of critical illness, and developed a clinical tool with these 10 variables to predict the probability of developing critical illness for COVID-19 patients [16]. The tool had a good performance of AUC (0.88). Yan and his colleagues used LDH, lymphocytes and high-sensitivity CRP to develop a model to identify patients at high risk of death [17]. Liu reported that the severity of the patients 
a

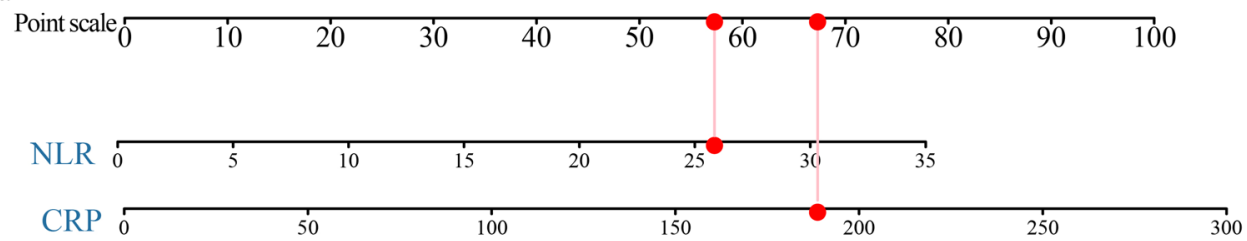

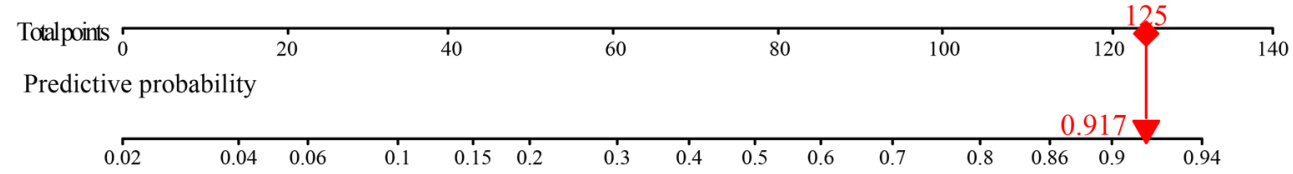

b
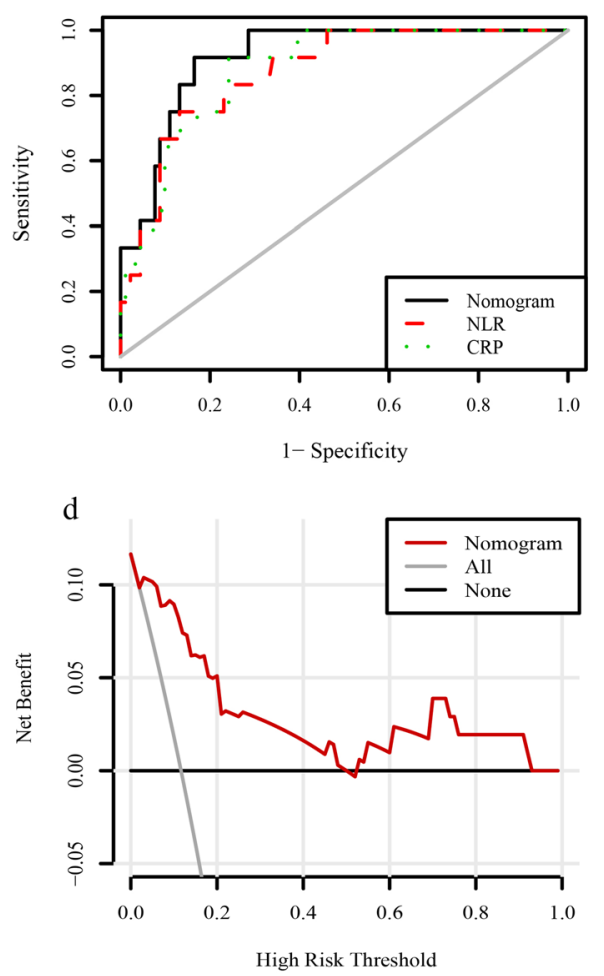

C

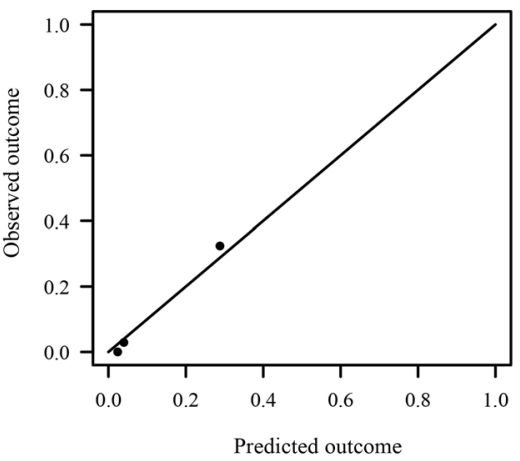

e

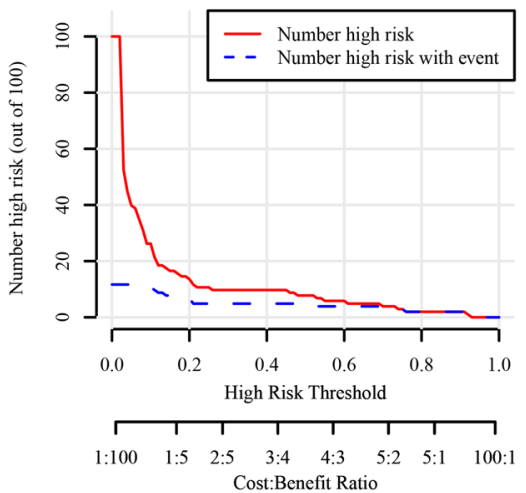

Fig. 1 Development and validation of a nomogram. a A nomogram for predicting the probability of death. The observed value of each variable could get a matching point according to the point scale. Use the total points of all variables to evaluate the probability of death. $\mathbf{b}$ ROC curves for the nomogram, NLR and CRP. c Calibration plot, d decision curve and e clinical impact curve for the nomogram. ROC, receiver operating characteristic; NLR, neutrophil-to-lymphocyte ratio; CRP, C-reactive protein

with COVID-19 could be predicted by age and NLR [18]. In this study, we found that NLR and CRP were the most related risk factors with death outcome. A nomogram for predicting the probability of death based on these two factors was developed, with a high AUC of 0.918 and clinical benefit. An algorithm based on the nomogram (as presented in Figs. 1a and 2) could help physicians to screen high death risk COVID-19 patients with cancer on admission, and give effective preventive measures or intensive care. The cut-off value of NLR and CRP were 7.90 and 34.62, respectively. The patients with NLR $<7.90$ and CRP $<34.62$ had no risk of dying. The mortality risk of patients with NLR $>7.90$ / CRP $<34.62$ or NLR $>7.90$ / CRP $>34.62$ was $16.7 \%$. However, more than $50 \%$ patients 


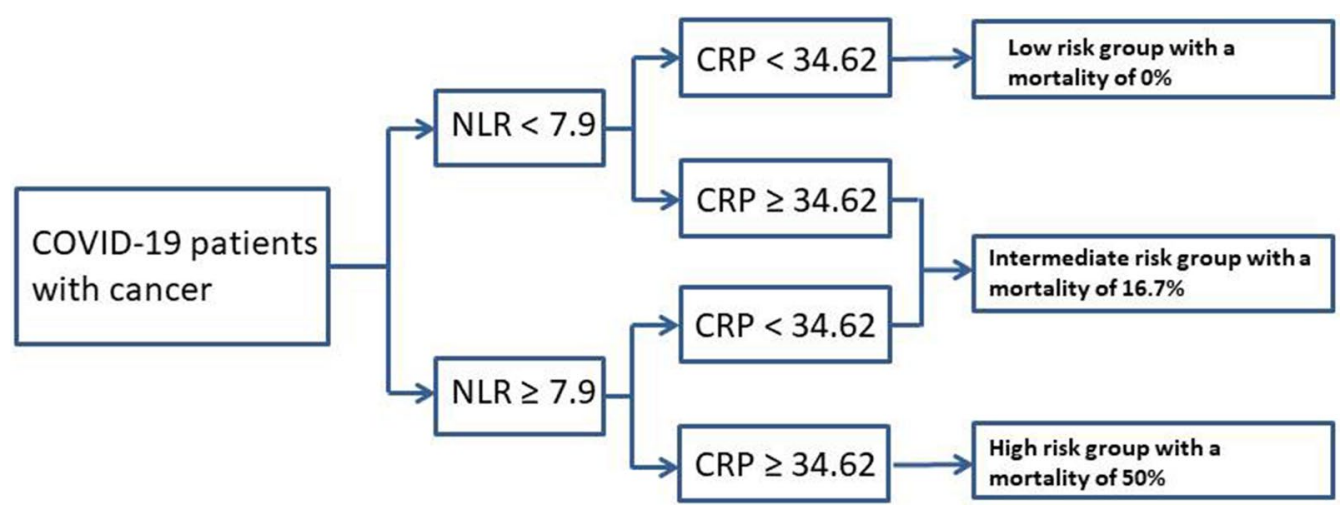

Fig. 2 A chart of risk stratification by using neutrophil-to-lymphocyte ratio and C-reactive protein

with NLR $>7.90$ and CRP $>34.62$ died (Fig. 2). The risk stratification would help us to take care of the high-risk patients and reduce the mortality.

There were several limitations in this study. First, the clinical records were incomplete. Especially no dynamic changes of laboratory results were recorded, which might help us to better identify severity or death outcome related risk factors. Second, the sample of COVID-19 patient with cancer was limited. No external validation of the nomogram was performed.

\section{Conclusion}

This study presented the details of clinical features and laboratory results in 103 COVID-19 patients with cancer. The main cancer categories were breast cancer, lung cancer, bladder cancer. The mortality was $11.7 \%$. In addition, this study found that NLR and CRP were death risk factors. An algorithm based on the two factors could help to screen high risk patients and give adequate treatment and protective measures in advance.

\section{Abbreviations}

COVID-19: Coronavirus disease 2019; AUC: Area under the curve; ROC: Receiver operating characteristic; IQR: Interquartile range; WBC: White blood cell; NLR: Neutrophil-to-lymphocyte ratio; CRP: C-reactive protein; IL-6: Interleukin-6; LDH: Lactate dehydrogenase; aHBDH: Alpha-hydroxybutyric dehydrogenase NTproBNP: N-terminal pro brain natriuretic peptide; AST: Aspartate transaminase; ALT: Alanine transaminase.

\section{Supplementary Information}

The online version contains supplementary material available at https://doi. org/10.1186/s12879-021-06495-9.

Additional file 1: Figure S1. A flow chart of patients selection.

\section{Acknowledgements}

This study was supported by the Research Projects of the Joint Logistics Support Force of PLA (TKTJKY2020003, TKTJKY2020029 and TKTJKY2020136).

\section{Authors' contributions}

JS, HW, ZX and QL designed the study. XW, XH, YF, BL, JL, GY and LL1 participated in data selection and assembly. XY, BW, SZ and LL2 performed the data analysis. YZ, QY and JY were involved in drafting the manuscript. YZ, QY and JS revised the manuscript critically for important intellectual content. All authors read and approved the final manuscript.

Funding

None.

Availability of data and materials

The datasets used and/or analysed during the current study are available from the corresponding author on reasonable request.

\section{Declarations}

\section{Ethics approval and consent to participate}

This study was approved by the ethics committee of the Ethics Committee of the Taikang Hospital (TKTJLL-007). The Ethics Committee of the Taikang Hospital waived the need for informed consent of each patient.

\section{Consent for publication}

Not applicable.

\section{Competing interests}

All the authors declared no potential conflicts of interest in this article.

\section{Author details}

${ }^{1}$ Cancer Institute, Xinqiao Hospital, Army Medical University, 83 Xinqiao Zhengjie Road, Shapingba, Chongqing 400037, People's Republic of China. ${ }^{2}$ Department of Ultrasound, The 941st Hospital of the PLA Joint Logistic Support Force, Xining, People's Republic of China. ${ }^{3}$ Department of Gastroenterology, Southwest Hospital, Army Medical University, Chongqing, People's Republic of China. ${ }^{4}$ Department of Emergency, Xinqiao Hospital, Army Medical University, Chongqing, People's Republic of China. ${ }^{5}$ Department of Neurology, Southwest Hospital, Army Medical University, Chongqing, People's Republic of China. ${ }^{6}$ Department of Hematology, Xinqiao Hospital, Army Medical University, Chongqing, People's Republic of China. ${ }^{7}$ Department of Respiratory and Intensive Care, Xinqiao Hospital, Army Medical University, 83 Xingiao Zhengjie Road, Shapingba, Chongqing 400037, People's Republic of China. ${ }^{8}$ Institute of Infectious Diseases, Southwest Hospital, Army Medical University, Chongqing, People's Republic of China. ${ }^{9}$ Department of Respiratory Medicine, Army Medical Center, Chongqing, People's Republic of China. ${ }^{10}$ Xinqiao Hospital, Army Medical University, 83 Xinqiao Zhengjie Road, Shapingba, Chongqing 400037, People's Republic of China. 
Received: 6 January 2021 Accepted: 29 July 2021

Published online: 05 August 2021

\section{References}

1. Zhou F, Yu T, Du R, et al. Clinical course and risk factors for mortality of adult inpatients with COVID-19 in Wuhan, China: a retrospective cohort study. Lancet. 2020;395:1054-62.

2. Tian S, Hu N, Lou J, et al. Characteristics of COVID-19 infection in Beijing. J Infect. 2020:80:401-6.

3. Orioli L, Hermans MP, Thissen JP, et al. COVID-19 in diabetic patients: related risks and specifics of management. Ann Endocrinol (Paris). 2020;81:101-9.

4. Schiffrin EL, Flack JM, Ito S, Muntner P, Webb RC. Hypertension and COVID-19. Am J Hypertens. 2020;33:373-4.

5. Guzik TJ, Mohiddin SA, Dimarco A, et al. COVID-19 and the cardiovascular system: implications for risk assessment, diagnosis, and treatment options. Cardiovasc Res. 2020;116:1666-87.

6. Liang W, Guan W, Chen R, et al. Cancer patients in SARS-CoV-2 infection: a nationwide analysis in China. Lancet Oncol. 2020;21:335-7.

7. Patel R, Park J, Shah A, Saif MW. COVID-19 and cancer patients. Cancer Med J. 2020;3:40-8.

8. Menetrier-Caux C, Ray-Coquard I, Blay JY, Caux C. Lymphopenia in cancer patients and its effects on response to immunotherapy: an opportunity for combination with cytokines? J Immunother Cancer. 2019;7:85.

9. Tian J, Yuan $X$, Xiao J, et al. Clinical characteristics and risk factors associated with COVID-19 disease severity in patients with cancer in Wuhan, China: a multicentre, retrospective, cohort study. Lancet Oncol. 2020;21:893-903
10. Yang F, Shi S, Zhu J, et al. Clinical characteristics and outcomes of cancer patients with COVID-19. J Med Virol. 2020;92:2067-73.

11. Cucinotta D, Vanelli M. WHO declares COVID-19 a pandemic. Acta Biomed. 2020;91:157-60.

12. Dai M, Liu D, Liu M, et al. Patients with cancer appear more vulnerable to SARS-CoV-2: a multicenter study during the COVID-19 outbreak. Cancer Discov. 2020;10:783-91.

13. Wang D, Hu B, Hu C, et al. Clinical characteristics of 138 hospitalized patients with 2019 novel coronavirus-infected pneumonia in Wuhan, China. JAMA. 2020;323:1061-9.

14. Wu Z, McGoogan JM. Characteristics of and important lessons from the coronavirus disease 2019 (COVID-19) outbreak in China: summary of a report of 72314 cases from the Chinese center for disease control and prevention. JAMA. 2020:323:1239-42.

15. Zhang L, Zhu F, Xie L, et al. Clinical characteristics of COVID-19-infected cancer patients: a retrospective case study in three hospitals within Wuhan, China. Ann Oncol. 2020;31:894-901.

16. Liang W, Liang $\mathrm{H}$, Ou L, et al. Development and validation of a clinical risk score to predict the occurrence of critical illness in hospitalized patients with COVID-19. JAMA Intern Med. 2020;180:1081-9.

17. Yan L, Zhang H, Goncalves J, et al. An interpretable mortality prediction model for COVID-19 patients. Nat Machine Intell. 2020;2:283-8.

18. Liu J, Liu Y, Xiang P, et al. Neutrophil-to-lymphocyte ratio predicts critical illness patients with 2019 coronavirus disease in the early stage. J Transl Med. 2020;18:206.

\section{Publisher's Note}

Springer Nature remains neutral with regard to jurisdictional claims in published maps and institutional affiliations.
Ready to submit your research? Choose BMC and benefit from:

- fast, convenient online submission

- thorough peer review by experienced researchers in your field

- rapid publication on acceptance

- support for research data, including large and complex data types

- gold Open Access which fosters wider collaboration and increased citations

- maximum visibility for your research: over $100 \mathrm{M}$ website views per year

At $\mathrm{BMC}$, research is always in progress.

Learn more biomedcentral.com/submissions 